Results: Excluded 40 patients with previous diagnosed dementia, total 5,496 gout patients were compared to 27,480 age- and sex-matched control group. The analysis results showed that gout patients had a lower risk of overall dementia [odds ratio (OR): 0.79; 95\% confidence interval $(\mathrm{Cl})$ : 0.63-0.99; $p<0.05$ ] after adjusted for age, sex, household income, comorbidities. The risk of $\mathrm{VaD}$ (OR: $0.77 ; 95 \% \mathrm{Cl}: 0.32-1.9 ; \mathrm{p}=0.68$ ) and $\mathrm{AD}(\mathrm{OR} ; 0.8 ; 95 \% \mathrm{Cl}: 0.53-1.19 ; \mathrm{p}=0.06$ ) tended to be lower in gout group but these were not significant.

Conclusion: Gout patients had a lower risk of dementia. This result tended to exist for both $\mathrm{AD}$ and $\mathrm{VaD}$ type of dementia.

REFERENCES:

[1] Hooper DC, Spitsin S, Kean RB, et al. Uric acid, a natural scavenger of peroxynitrite, in experimental allergic encephalomyelitis and multiple sclerosis. Proc Natl Acad Sci USA. 1998; 95: 675-80.

[2] Bowman GL, Shannon J, Frei B, et al. Uric acid as a CNS antioxidant. J Alzheimer's Dis. 2010; 19: 1331-6.

Disclosure of Interests: None declared

DOI: 10.1136/annrheumdis-2021-eular.2292

\section{OP0208 INCIDENT GOUT: RISK OF DEATH AND CAUSE- SPECIFIC MORTALITY IN WESTERN SWEDEN - A NESTED CASE CONTROL STUDY}

M. Dehlin ${ }^{1}$, T. Zverkova Sandström ${ }^{1}$, L. T. H. Jacobsson ${ }^{1} .{ }^{1}$ Institute of Medicine, Sahlgrenska Academy, Department of Rheumatology and Inflammation Research, Gothenburg, Sweden

Background: Excess mortality in gout has been attributed to it's positive association with many comorbidities. Lately, a negative association with dementia has been suggested in some cohort studies. Data is limited on how this reflects in cause-specific mortality of gout patients.

Objectives: Our aims were to determine the relative risks in incident gout patients compared to general population 1) for overall death 2) for cause-specific mortality.

Methods: All incident cases of gout (definition: $1 \geq$ ICD-10 code for gout (M10/ M14.0) in the population based health care database VEGA; no gout diagnosis $\geq 5$ years previously) between 2006-2015 in western Sweden were identified. Five population controls per case matched on age, sex and place of residence were identified. Comorbidities, defined by ICD-10 codes, (alcohol related disorders, hypertension, ischemic heart disease, heart failure, cerebrovascular disease, diabetes, dyslipidemia, obesity, renal disease, dementia, lung diseases and cancer) were identified for 5 years preceding index date for cases and controls in VEGA. Patients were followed until the earliest of death, migration or study end 31 of December 2017. Cause of death was identified through the national cause of death register and grouped as death due to: CVD, renal disease, dementia, infections, diabetes, diseases of the digestive system, lung diseases, cancer and other. COX regression analyses, adjusted for baseline demographics and comorbidities, were used to compare mortality in cases and controls, taking competing cause of death into account by the Fine and Gray method.

Results: From the total adult population of western Sweden between 2006 and 2015 (yearly average of 1264150 individuals), we identified 24320 cases of incident gout and 119781 controls, who were followed on average 5.3 and 5.6 years respectively. At baseline mean age was (SD) 67.3 years (15.3) and $67.6 \%$ were men. At baseline, income and level of education was significantly lower in gout cases compared to controls, whereas all comorbidities were significantly more

Table 1. Causes of death by exposure status and cause-specific mortality overall, gout cases compared to controls, adjusted hazard ratios by the Fine and Gray method, $95 \%$ confidence intervals

\begin{tabular}{lcccc}
\hline Causes of death & \multicolumn{2}{c}{ Cause of death, $\mathrm{n}(\%)$} & \multicolumn{2}{c}{ Hazard ratios $(95 \% \mathrm{Cl})$} \\
\cline { 2 - 5 } & $\begin{array}{c}\text { Gout cases, } \mathrm{n} \\
\mathrm{n} 24320\end{array}$ & $\begin{array}{c}\text { Controls, } \mathrm{n}= \\
119781\end{array}$ & Model 1 & Model 2 \\
& $3527(14.5)$ & $11369(9.5)$ & $1.61(1.55-1.67)$ & $1.20(1.16-1.26)$ \\
& $140(0.6)$ & $239(0.2)$ & $2.79(2.26-3.44)$ & $1.68(1.31-2.16)$ \\
Cardiovascular disease & $287(1.2)$ & $2922(2.4)$ & $0.45(0.40-0.51)$ & 0.68 \\
Renal disease & & & & $(0.60-0.77)$ \\
Dementia & $437(1.8)$ & $1631(1.4)$ & $1.27(1.14-1.41)$ & $1.15(1.03-1.28)$ \\
& $250(1.0)$ & $665(0.6)$ & $1.80(1.55-2.08)$ & $1.02(0.87-1.20)$ \\
Infections & $226(0.9)$ & $725(0.6)$ & $1.49(1.29-1.74)$ & $1.36(1.16-1.59)$ \\
Diabetes & $306(1.3)$ & $1216(1.0)$ & $1.20(1.06-1.36)$ & $0.89(0.78-1.01)$ \\
Diseases digestive system & $1179(4.9)$ & $6067(5.1)$ & $0.92(0.87-0.98)$ & 0.94 \\
Lung diseases & & & & $(0.88-0.99)$ \\
Cancer & $655(2.7)$ & $2899(2.4)$ & $1.08(0.99-1.17)$ & $1.09(0.99-1.19)$ \\
& $7007(28.8)$ & $27733(23.2)$ & $1.32(1.29-1.36)$ & $1.08(1.05-1.11)$ \\
Other & & & &
\end{tabular}

Model 1: adjusted for age and sex

Model 2: adjusted for baseline values for: age, sex, marital status, income, education, born outside of Sweden, alcohol related disorders, hypertension, ischemic heart disease, heart failure, cerebrovascular disease, diabetes, dyslipidemia, obesity, renal disease, dementia, lung diseases and cancer common among gout cases (data not shown) except for dementia which was significantly more common in controls, $4.2 \%$ versus $2.5 \%$ in cases, $p<.0001$. The HR for overall death was increased in gout patients (HR: 1.08 (95\% CL: 1.05-1.11) (Table 1). Gout cases had significantly increased risk for death by CVD, rena disease, infections, diseases of the digestive system and significantly decreased risk for death by dementia and cancer (Table 1). Point estimates were similar in men and women (data not shown).

Conclusion: This study demonstrates a modest increase in overall mortality in gout patients and a decreased risk of death from dementia reflecting it's inverse relation to gout.

Disclosure of Interests: None declared

DOI: 10.1136/annrheumdis-2021-eular.454

\section{\begin{tabular}{|l|l}
\hline OP0209 SONOGRAPHIC ESTIMATION OF MONOSODIUM \\
\hline
\end{tabular} URATE BURDEN PREDICTS THE FULFILMENT OF THE 2016 REMISSION CRITERIA FOR GOUT: A 12-MONTH STUDY}

E. Cipolletta ${ }^{1}$, A. DI Matteo ${ }^{1}$, W. Grassi ${ }^{1}$, E. Filippucci ${ }^{1} .{ }^{1}$ Polytechnic University of Marche, Department of Clinical and Molecular Sciences, Ancona, Italy

Background: Preliminary remission criteria for gout include the following five domains: serum uric acid (SUA) levels $<360 \mu \mathrm{mol} / \mathrm{l}$, absence of subcutaneous tophi, absence of gouty flares, pain (due to gout) $<2 / 10$ and patient global assessment of disease activity $<2 / 10$ [1]. To achieve remission, all criteria must be fulfilled at least twice in a 12-month period [1]. Although imaging techniques allow to visualise monosodium urate (MSU) deposits and to estimate their burden, an imaging domain was not included.

Objectives: To investigate whether baseline MSU burden estimated by ultrasonography (US) predicts the achievement of remission according to these criteria [1] over 12 months.

Methods: In this 12-month prospective, observational and monocentric study, patients with gout according to the 2015 ACR/EULAR criteria and on urate-lowering therapy for at least the preceding 6 months were consecutively recruited. After the baseline clinical assessment, only patients fulfilling all domains of the remission criteria [1] (and therefore amenable to achieve the remission at 12 months) were followed up at 6-month intervals for one year.

The US findings indicative of MSU deposits were identified according to the OMERACT definitions. The following joints were included in the US scanning protocol: elbows, wrists, $2^{\text {nd }}$ metacarpophalangeal joints, knees, ankles and $1^{\text {st }}$ metatarsophalangeal joints. Sum scores of aggregates, double contour (DC) signs, and tophi were calculated separately, and a total score resulting from the sum of all elementary US findings was recorded.

Results: Seventy patients with gout were recruited; of these, $20(28.6 \%)$ were excluded at baseline because $13(18.6 \%)$ did not satisfy the SUA domain, $11(15.7 \%)$ tophus domain, 7 (10.0\%) pain domain and $5(7.1 \%)$ disease activity domain

In the enrolled patients (Age: $59.9 \pm 14.8$ years, female/male ratio: $1 / 49$, disease duration: $6.5 \pm 6.6$ years), remission criteria were fulfilled in $21(42.0 \%)$ of 50 patients over 12 months.

No significant difference was found between patients fulfilling and not fulfilling the remission criteria at 12 months in all clinical and laboratory data except for the gout flare prophylaxis $(7,33.3 \%$ vs $20,69.0 \%$; $p=0.02)$.

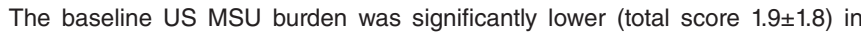
patients in remission than in those not in remission at 12 months (total score $5.1 \pm 3.1)(p<0.01)$. The fulfilment of the remission criteria at 12 months was recorded in $87.5 \%$ of the patients without baseline US evidence of MSU deposits and in only $33.0 \%$ of those with at least one US finding indicating MSU deposits $(\mathrm{p}<0.01)$.

US scores and gout flare prophylaxis were the only significant predictors of remission in the univariate analyses (Table 1)

Table 1. Predictive values of baseline data for the remission at 12 months.

\begin{tabular}{lcc}
\hline & OR $(\mathbf{9 5 \%} \% \mathbf{C l})$ & P value \\
\hline Ongoing flare prophylaxis & $0.23(0.07-0.75)$ & $\mathbf{0 . 0 2}$ \\
Total score $=0$ & $10.83(1.14-102.59)$ & $\mathbf{0 . 0 4}$ \\
Aggregate score $=0$ & $5.53(1.34-22.76)$ & $<0.01$ \\
DC sign score $=0$ & $7.33(1.71-31.44)$ & $<0.01$ \\
Tophus score=0 & $3.88(1.08-13.92)$ & $\mathbf{0 . 0 2}$ \\
\hline
\end{tabular}

95\% Cl: 95\% confidence interval; OR: odds ratio.

The risk of not fulfilling the remission criteria increased with the US burden of MSU deposits. For each 1-point increase in total score, aggregate score, DC sign score and tophus score, the risk increased by $1.81-(95 \% \mathrm{Cl}: 1.27-2.60), 1.73-$ (95\%Cl: $1.14-2.64), 4.16-(95 \% \mathrm{Cl}: 1.55-11.3)$ and 1.95 -fold $(95 \% \mathrm{Cl}: 1.07-3.56)$ respectively (Figure 1) 


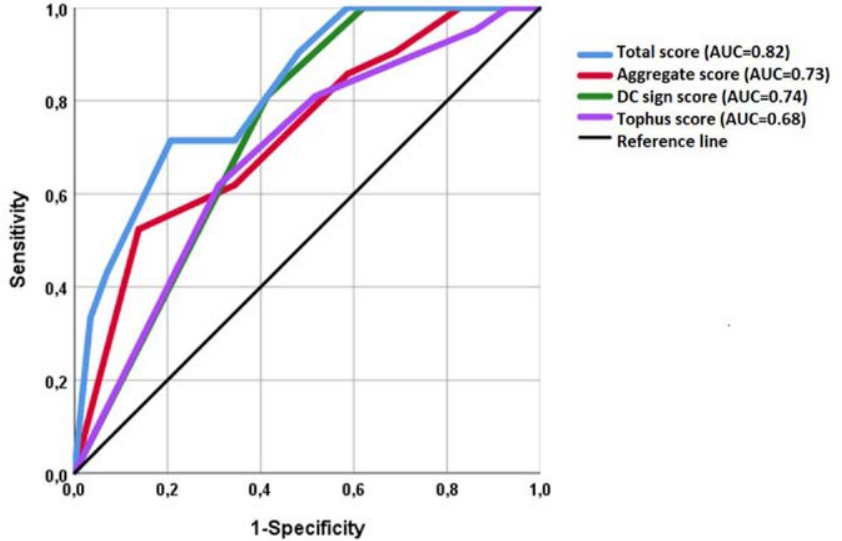

Conclusion: Baseline US estimation of MSU burden is an independent predictor of gout clinical remission at 12 months. The absence of US MSU deposits was the most significant predictor of remission, whereas the US detection of DC sign in at least one joint of not achieving remission. Thus, performing an US examination in patients amenable to fulfil the remission criteria after 12 months may improve risk-stratification and inform management of these patients.

\section{REFERENCES:}

[1] de Lautour H, et al. Development of Preliminary Remission Criteria for Gout Using Delphi and 1000Minds Consensus Excersices. Arthritis Care Res 2016;68:667-72

Disclosure of Interests: Edoardo Cipolletta: None declared, Andrea Di Matteo: None declared, Walter Grassi Speakers bureau: Walter Grassi has received speaking fees from AbbVie, Celgene, Grünenthal, Pfizer and Union Chimique Belge Pharma., Emilio Filippucci Speakers bureau: Emilio Filippucci. has received speaking fees from AbbVie, Bristol-Myers Squibb, Janssen-Cilag, Novartis, Pfizer, Roche and Union Chimique Belge Pharma DOI: 10.1136/annrheumdis-2021-eular.495

\section{Rheumatoid arthritis - comorbidity and clinical aspects - II}

\section{OP0210 PREGNANCY OUTCOMES IN RELATION TO DISEASE ACTIVITY AND ANTI-RHEUMATIC TREATMENT STRATEGIES IN WOMEN WITH RHEUMATOID ARTHRITIS - A MATCHED COHORT STUDY FROM SWEDEN AND DENMARK}

K. Hellgren ${ }^{1,2}$, A. E. Secher ${ }^{3}$, B. Glintborg ${ }^{3}$, A. Lilleoere Rom ${ }^{4}$ B. Gudbjornsson ${ }^{5}$, B. Michelsen ${ }^{6}$, F. Granath ${ }^{1}$, M. L. Hetland ${ }^{3} .{ }^{1}$ Karolinska Institutet, Clinical Epidemiology Division, Department of Medicine Solna, Stockholm, Sweden; ${ }^{2}$ Karolinska Institutet, Division of Rheumatology, Department of Medicine Solna, Stockkholm, Sweden; ${ }^{3}$ DANBIO and COPECARE, Center for Rheumatology and Spine Diseases, Centre of Head and Orthopedics, Rigshospitalet, Copenhagen, Denmark; ${ }^{4}$ Rigshospitalet, Department of Obstetrics and Research Unit Women's and Children's Health, Copenhagen, Denmark; ${ }^{5}$ Landspitali University Hospital and University of Iceland, Centre for Rheumatology Research and Faculty of Medicine, Reykjavik, Iceland; ' Diakonhjemmet Hospital, Department of Rheumatology, Oslo, Norway

Background: Women with rheumatoid arthritis (RA) are at increased risks of adverse pregnancy outcomes, especially preterm birth (PTB) and small for gestational age (SGA). However, the link between RA disease activity, type and timing of anti-rheumatic treatment, and the risk of these outcomes remains unclear. Objectives: To explore the associations between maternal RA and PTB/SGA in relation to disease activity and use of anti-rheumatic treatment before and during pregnancy.

Methods: By linking national medical birth registers to prospective clinical rheumatology registers (CRRs) in Sweden (SRQ) and Denmark (DANBIO), we identified 1739 RA-pregnancies and 17390 control-pregnancies (matched 1:10 on maternal age, birth year, and parity) with delivery 2006-2018. From CRRs and prescribed drug registers, we collected information on RA disease activity (DAS28, CRP and HAQ-score) and anti-rheumatic drugs (biologics, conventional synthetic (cs)DMARDs and oral steroids) nine months before and during pregnancy. Using logistic regression, we estimated adjusted odds ratios (ORs) with $95 \%$ confidence intervals (CI) for PTB and SGA in RA-pregnancies vs. control-pregnancies overall, and stratified by disease activity and type of anti-rheumatic treatment before and during pregnancy. Apart from the matching variables we adjusted for body mass index, smoking, educational level and country.

Results: Overall, RA-pregnancies were associated with increased ORs of PTB (1.92, 95\% Cl 1.56-2.35) and SGA (1.93, 95\% Cl 1.45-2.57). High maternal disease activity during pregnancy strengthened the associations with both PTB and SGA, whereas the ORs approached 1 for low disease activity (control-pregnancies constituting the reference), Table 1. Among RA-pregnancies with available information on DAS28-CRP $(\mathrm{n}=686,39 \%)$, OR was $2.69(95 \% \mathrm{Cl} 1.37-5.26)$ for PTB, and 3.39 (95\% Cl 1.43-8.06) for SGA, comparing DAS28-CRP >=3.2 vs. $<3.2$ during pregnancy. Stratifying on type of anti-rheumatic treatment did not substantially change the results. Combination therapy with biologics together with oral steroids and/or csDMARDs in the nine months before pregnancy was associated with PTB (ORs spanning 2.57-3.45) and SGA (ORs spanning 2.40-3.81). Conclusion: During pregnancy, disease activity rather than treatment, appears to be the most important risk factor for PTB and SGA in RA. The findings highlight the importance of monitoring RA during pregnancy, especially in women receiving extensive anti-rheumatic treatment or with residual disease activity.

Acknowledgements: The Nordic clinical rheumatology registers for allowing us to use their clinical data. We also would like to acknowledge the NordForsk and FOREUM, especially the patient representatives of the NordForsk collaboration. Disclosure of Interests: Karin Hellgren Consultant of: UCB, Anne Emilie Secher None declared, Bente Glintborg Grant/research support from: Pfizer, Biogen and BMS, Ane Lilleoere Rom: None declared, Björn Gudbjornsson Speakers bureau: Amgen and Novartis, Brigitte Michelsen Consultant of: Novartis, Grant/ research support from: Novartis, Fredrik Granath: None declared, Merete Lund Hetland Speakers bureau: Biogen, Celltrion, Janssen Biologics B.V, MSD, Pfizer, Samsung Biopis, Consultant of: Biogen, Celltrion, Janssen Biologics B.V, MSD, Pfizer, Samsung Biopis, Grant/research support from: AbbVie, Biogen, BMS, Eli Lilly Danmark A/S, Lundbeck Fonden, Pfizer, Roche, Sandoz, Novartis DOI: 10.1136/annrheumdis-2021-eular.605

Table 1. Adjusted odds ratios (ORs)1 for PTB and SGA in RA-pregnancies in relation to disease activity and functional status during pregnancy vs. control pregnancies

\begin{tabular}{|c|c|c|c|c|c|c|c|}
\hline & & \multicolumn{3}{|c|}{ Preterm birth } & \multicolumn{3}{|c|}{ Small for gestational age ${ }^{1}$} \\
\hline & & Pregnancies, $n$ & $\begin{array}{c}\text { Events, } \\
\mathrm{n}(\%)\end{array}$ & $\begin{array}{l}\text { Adjusted OR } \\
\qquad(95 \% \mathrm{Cl})\end{array}$ & Pregnancies, $\mathrm{n}$ & $\begin{array}{c}\text { Events, } \\
\mathrm{n}(\%)\end{array}$ & $\begin{array}{l}\text { Adjusted OR } \\
\qquad(95 \% \mathrm{Cl})\end{array}$ \\
\hline \multicolumn{2}{|l|}{ Control-pregnancies $^{2}$} & 17312 & $794(5)$ & 1 (REF) & 17184 & $418(2)$ & 1(REF) \\
\hline & 1734 & $144(8)$ & $1.92(1.56-2.35)$ & 1722 & $75(4)$ & $1.93(1.45-2.57)$ \\
\hline \multirow[t]{4}{*}{ DAS28-CRP ${ }^{3,4}$} & $<3.2$ & 459 & $26(6)$ & $1.05(0.64-1.72)$ & 456 & $13(3)$ & $0.96(0.49-1.91)$ \\
\hline & $3.2-5.1$ & 182 & $17(9)$ & $2.40(1.40-4.11)$ & 181 & $13(7)$ & $3.13(1.64-5.97)$ \\
\hline & $>5.1$ & 43 & $5(12)$ & $2.77(0.86-8.87)$ & 43 & $4(9)$ & 4.59 (1.59-13.2) \\
\hline & No information & 1050 & $96(9)$ & $2.18(1.71-2.78)$ & 1042 & $45(4)$ & $2.06(1.46-2.90)$ \\
\hline \multirow[t]{4}{*}{$\mathrm{HAQ}$-score ${ }^{3}$} & $<0.5$ & 338 & $19(6)$ & $1.31(0.79-2.16)$ & 335 & $8(2)$ & $0.93(0.41-2.12)$ \\
\hline & $0.5-0.9$ & 166 & $15(9)$ & $2.37(1.34-4.19)$ & 165 & $5(3)$ & $1.50(0.60-3.74)$ \\
\hline & $\geq 1$ & 196 & $19(10)$ & $1.85(1.06-3.24)$ & 195 & $18(9)$ & $3.70(2.05-6.67)$ \\
\hline & No information & 1034 & $91(9)$ & $2.06(1.60-2.64)$ & 1027 & $44(4)$ & $1.98(1.39-2.82)$ \\
\hline \multirow[t]{4}{*}{$\mathrm{CRP}, \mathrm{mg} / \mathrm{L}^{3}$} & $<10$ & 455 & $21(5)$ & $0.91(0.55-1.51)$ & 452 & $14(3)$ & $1.09(0.57-2.07)$ \\
\hline & $10-29$ & 191 & $22(11)$ & $2.58(1.52-4.38)$ & 190 & $12(6)$ & $2.68(1.38-5.22)$ \\
\hline & $\geq 30$ & 57 & $9(16)$ & $4.59(2.28-9.22)$ & 57 & $5(9)$ & $4.12(1.68-10.1)$ \\
\hline & No information & 1031 & $92(9)$ & $2.10(1.64-2.70)$ & 1023 & $44(4)$ & $2.05(1.44-2.90)$ \\
\hline
\end{tabular}

${ }^{1}$ Missingness on small for gestational age in 12 RA-pregnancies and 128 control-pregnancies ${ }^{2}$ Only among live births, i.e. stillbirths excluded. ${ }^{3}$ Maximum value any time during pregnancy ${ }^{4}$ Defined as DAS28-CRP without patient's global health VAS 